\title{
Root resection for future implant site development - the transitional approach
}

\begin{abstract}
In every clinical situation there are a wide variety of treatment options that can be undertaken. As practitioners we owe our patients to be able to provide a wide range of treatment options based on, the clinical situation, age, economical considerations of the patient, and the best available clinical evidence of successful treatment modality. Patients are also becoming more educated in the available dental treatments and demands for conservative approach. This paper offers the treatment approach that preserves tooth structure in cases that are carefully selected. The molar tooth with extensive decay affected by endo-perio combined disease threatens the loss of the tooth. Hemi-section of the effected tooth allows the preservation of tooth structure, alveolar bone and cost savings (time and money) over other treatment options. In the right situation a fine restorative result can easily be attained using the root-section option.
\end{abstract}

Volume 9 Issue 2 - 2018

\section{Ankit Jivan Desai, Sohini Choudhary, Triveni M Gowda, DS Mehta}

Department of Periodontics, Bapuji Dental College and Hospital, India

\author{
Correspondence: Ankit Jivan Desai, Department Of \\ Periodontics, Bapuji Dental College and Hospital, Davangere- \\ 577004 Karnataka, India, Tel +91 8141040950,
} Email drankitd@gmail.com

Received: February 09, 2017| Published: April 05, 2018

Keywords: Furcation Involvement, Hemisection, Root Resection, Socket Preservation, Implant Site Development

\section{Introduction}

The loss of posterior molar can result is several undesirable sequelae including migration of teeth, collapse of the vertical dimension of occlusion, super eruption of opposing teeth, loss of supporting alveolar bone and a decrease in chewing ability. Mandibular first molar is of major importance because of its role in occlusion. Extensive caries and/or advanced furcation involvement of mandibular molars have always been a therapeutic challenge for clinicians. Lack of conservative approaches to such lesions questions long-term retention of the tooth \& thus the hopeless prognosis suggest extraction as the only treatment choice available. The treatment options to replace severely damaged and possibly unrestorable teeth include removable partial denture, fixed partial denture and dental implants. But patient's desire to maintain their natural dentition and financial reasons to avoid prosthetically driven implant therapy has made clinicians think twice before extraction of the tooth. Advances in dentistry have made it possible to maintain a functional dentition for a lifetime. Sectioning of a molar into two halves followed by removal of the diseased root and its coronal portion is referred as Hemisection. ${ }^{1}$ It is a conservative option with acceptable prognosis. ${ }^{2}$ The terms 'hemi-section' and 'root amputation' are known collectively as 'root resection'. ${ }^{3}$ The success rate of this kind of endoperio cases depends on anatomical characteristic of furcation area for adequate instrumentation and satisfactory bone support in relation to the healthy root. Proper case selection followed by interdisciplinary approach with endodontic, periodontic and prosthetic procedures can produce predictable results. ${ }^{2}$

Weine has listed the following indications \& contraindications for tooth resection. ${ }^{4}$

\section{Periodontal indications}

1. Severe vertical bone loss limited to one root of multi-rooted teeth

2. Through and through Grade-III type of advanced furcation involvement which cannot be corrected with periodontal procedures.
3. Unfavourable proximity of roots of adjacent teeth, preventing adequate hygiene maintenance in proximal areas.

4. Severe root exposure due to dehiscence

\section{Endodontic and restorative indications}

a. Failure of an abutment within a fixed prosthesis: If a singlerooted or multi-rooted tooth with vertical fracture, endodontic failure or periodontal involvement within a fixed bridge \& if the remaining abutment support is sufficient, the root of the involved tooth is extracted instead of removing the entire bridge.

b. Endodontic failure like large root perforation, or perforation through the floor of the pulp chamber,

c. Difficult to instrument pulp canal of one of the roots of an endodontically involved tooth

d. Vertical fracture of one root while the other roots are unaffected in a multi-rooted tooth.

e. Severe destructive process: Furcation or subgingival caries, traumatic injury, or external root resorption.

\section{Contraindications}

i. Strong abutment tooth adjacent to the proposed hemisection, which could act as an abutment to a fixed prosthesis.

ii. Poorly shaped roots or fused roots

iii. Inoperable root canal making endodontic procedure difficult

iv. Patient unwilling to undergo surgical and endodontic treatments.

\section{Case report}

A 31 year old male patient reported to the Bapuji Dental College and hospital, Davangere, Karnataka, India with the chief complaint of pain and food lodgement in lower left back tooth region since 5 months. Pain was dull aching and intermittent in nature, which 
aggravated on mastication. He also complains of occasional swelling $\&$ pus discharge in the same. On further enquiry, patient did not give any significant medical and previous dental history. Extra oral examination revealed no abnormality. On intraoral examination, extensive caries involving mesial half of the crown of 36 teeth and a chronic sinus tract were present (Figure 1). Probing depth was around 7-8 mm buccally and mesially with Grade-III furcation involvement. The tooth was tender on vertical percussion. IOPA radiograph of 36 revealed radiolucency in the mesio-occlusal portion of the crown portion extending into furcation area. IOPA showed grade III furcation defect with periodontal bone loss more along the mesial root as compared with distal root and also presence of periapical rarefaction with the mesial root (Figure 2). Periodontal support of distal root of 36 was good. Interproximal bone loss was seen between 35 and 36 . Vitality test was negative indicating pulp necrosis. Thus, it was diagnosed as "Combine Endodontic-Periodontal Lesion" in relation to 36 .

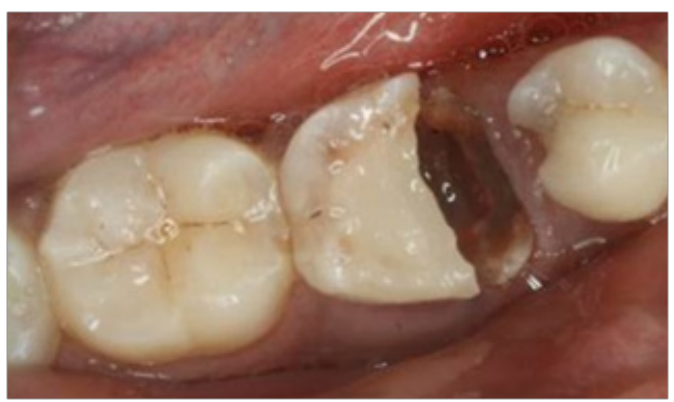

Figure I Fractured lower left molar.

\section{Treatment}

Patient was given the following options

a. Extraction with immediate guided gone regeneration (GBR) and delayed implant therapy

b. Hemisection with socket grafting and delayed implant therapy.

Patient insisted to save his natural tooth $\&$ also due to economic reasons, hemisection was decided as the final management. Patient was made understand the importance of socket grafting as the implant site development for future implant therapy. Whole procedure was explained to the patient $\&$ diagnostic impressions were made with irreversible hydrocolloid impression material.

\section{Endodontic phase: (Figure 2)}

Caries were removed \& root canal treatment was done in distal root of 36. After 7 days follow-up, Post-obturative restoration was performed using nano-hybrid composite material. Periodontal phase included thorough scaling and root planing. Gingival and periodontal status was reevaluated after 3 weeks.

\section{Periodontal phase: (Figure 3)}

The tooth was asymptomatic and thus root amputation was planned. After local anesthesia, a full thickness mucoperiosteal flap was reflected to expose the area of furcation. After flap reflection, bony defect was evident and debridement was done. A long shank tapered fissure carbide bur was used to make vertical cut facio-lingually towards the bifurcation area and hemisection was carried out (Figure 3A). Mesial root was extracted followed by thorough debrided and irrigation (Figure 3B). Odontoplasty was performed to contour the mesial aspect of distal root in such a way so as to facilitate oral hygiene measures. Pre-suturing was done with 3-0 silk non-resorbable suture $\&$ the socket was packed with bone graft (Novabone Putty, bioactive alloplastic graft) (Figure 3D). It was covered by PRF membrane. The flap was sutured in interrupted manner (Figure 3E). COE pack periodontal dressing was placed. Post-operative instructions were given. Antibiotics and analgesics were prescribed for 5 days. The sutures were removed 14 days later. The patient was monitored on a weekly schedule, postoperatively, to ensure good oral hygiene in the surgical area (Figure 3C). The surgical site was then allowed to heal with no occlusal stress on distal root for 4 weeks. On 1 month recall visit, healing was satisfactory without any mobility in relation to distal part of 36 . The fixed prosthesis was planned involving $35 \& 36$.

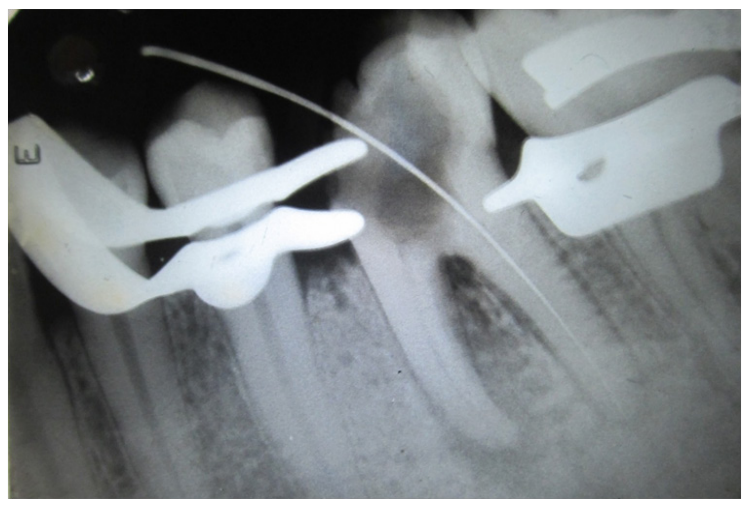

Figure 2 Endodontic therapy of distal root of 36 .

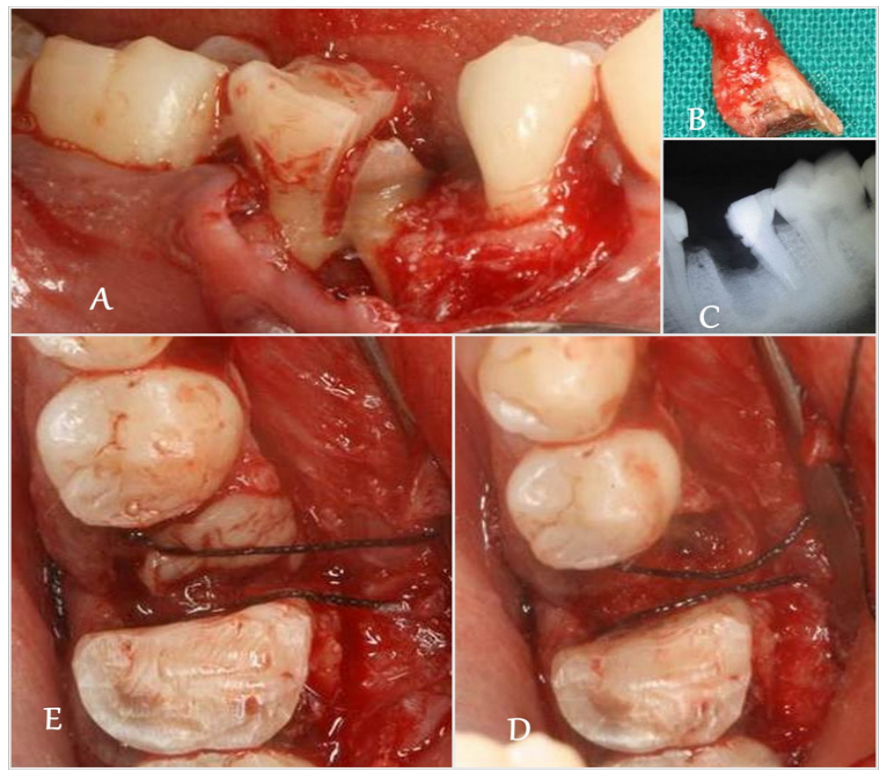

Figure 3 Periodontal surgical phase, A: Sectioning of the mesial root from furcation; B: Extracted mesial root piece; C: 14 days post-extraction X-ray image; D: Mesial socket preservation with bone grafting; F: PRF membrane placement \& suturing.

\section{Prosthetic phase: (Figure 4)}

Diagnostic impressions were made with irreversible hydrocolloid impression material and diagnostic casts were obtained. Face bow record was made and transferred to a semi-adjustable articulator and maxillary cast was mounted. Mandibular diagnostic cast was mounted 
using interocclusal record, to check for any occlusal prematurities and interferences and necessary occlusal corrections were carried out. Tooth preparation was done in relation to 35 , distal coronal aspect of 36 to receive 2- unit porcelain fused to metal full coverage restoration. Final impression was made using putty-reline technique and master cast was obtained. Mandibular master cast was mounted using interocclusal record. Wax pattern was fabricated, sprued, and invested. Casting procedure was carried out using standard techniques. Metal framework was tried in the patient's mouth followed by ceramic build up and bisque try in. Final prosthesis was cemented using glass ionomer cement. Post cementation instructions regarding periodontal maintenance were given. Occlusally, the tooth 36 was contoured as a molar on hemisected tooth, which provided more surface area for occlusal table (Figure 4A) (Figure 4B). Patient was followed up by regular recall visits on 4.5, 6, 9 and 12 months. 4.5 and 6 months IOPA revealed good bone regeneration which indicates good uptake of the graft. On 12 month recall, patient had good masticatory efficiency with the prosthesis and was very satisfied with the treatment outcome (Figure 4C).

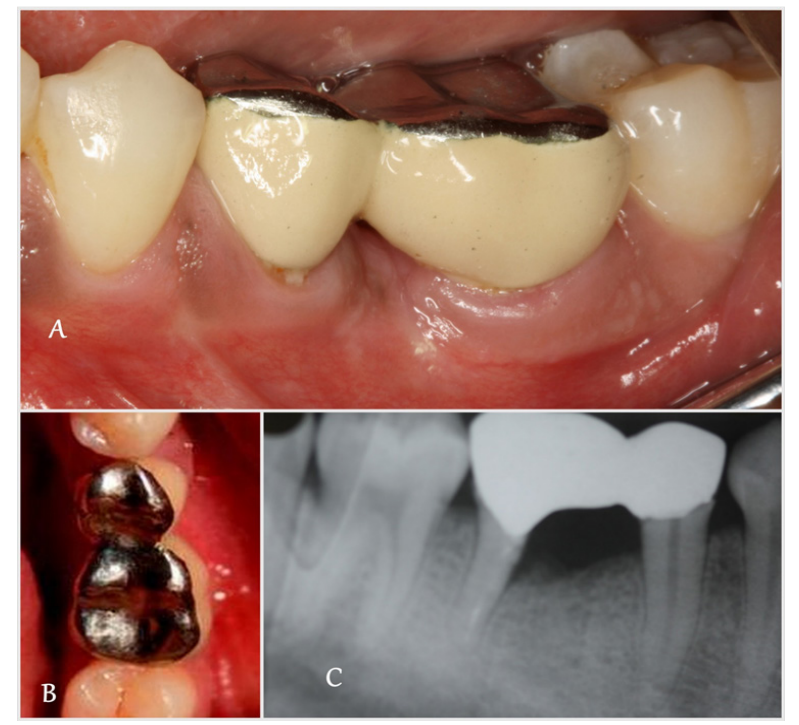

Figure 4 Prosthetic phase, A: Final bridge placement buccal view; B: Final bridge occlusal View; C: 12 Months post-therapy recall X-ray.

\section{Discussion}

The presence of patent accessory canals is a potential pathway for the spread of bacterial and toxic by-products, resulting in a direct inflammatory process in the periodontal ligament. If a primary endodontic disease remains untreated for a period of time, pulpitis may cause an inflammatory reaction in the interradicular periodontal tissues leading to secondary periodontal breakdown. Plaque forms at the gingival margin of the sinus tract, leads to localised plaqueinduced periodontitis in the area. And thus the lesion becomes combined type of endodontic-periodontal defect. Bone loss caused by only pulpal disease is reversible, whereas, advanced bone loss caused by periodontal or combined defects are usually irreversible. ${ }^{5}$ The treatment and prognosis of the primary endodontic disease with secondary periodontal involvement or a true combined lesion are different that those of teeth involved with only primary endododntic disease. The tooth now requires both endodontic and periodontal treatments because, with endodontic treatment alone, only part of the lesion will heal to the level of the secondary periodontal lesion.
If the endodontic treatment is adequate, the prognosis depends on the severity of the plaque-induced periodontitis and the efficacy of periodontal treatment. ${ }^{6}$ Here in the present case of 36 , caries involving pulp, periapical radiolucency on radiograph and the non-vital tooth with a sinus tract was a definite indication of endodontic treatment. But the caries were extending to the floor of the furcation with bone loss in furcation (Grade -III) and periapical region of mesial root of 36. Treatment options included extraction of 36 , followed by GBR $\&$ placement of implant, a fixed or a removable partial denture. The patient did not wish to have the tooth removed and go for expensive implant therapy. Hemisection is a suitable alternative to extraction. It aims to preserve as much natural tooth structure as possible. ${ }^{7}$ Buhler in 1994 stated that hemisection should be considered before every molar extraction as it provides a good absolute biological cost savings with good long term success. In addition, he reported that the failure rates of single-tooth alloplastic (titanium) implants and hemisections are not substantially different. ${ }^{8}$ Periodontal prognosis was fair as there was good bone support around the distal root of 36 . So conservative treatment was selected, which included hemisection of the mesial root of 36, followed by prosthetic replacement. Proper case selection and through endodontic, periodontal, restorative and maintenance therapies are the prerequisite for the success of the procedure. The extent and pattern of bone loss, root trunk and root length, ability to eliminate the osseous defects, mobility of the tooth and endodontic-restorative consideration, were all considered. ${ }^{9}$ Use of bone grafts and barrier membranes (GBR/GTR) can help to achieve regeneration by means of new attachment. Here in the present case, Novabone putty (bioactive Calcium-Phosphosilicate bone graft material) was used for socket preservation. CPS putty is a third-generation bioactive graft material that not only provides a physical scaffold for the bone tissue to grow (osteoconduction), but also interacts chemically with the surrounding tissue to impart an increased level of osteoblastic activity at the defect site (osteostimulation) for faster regeeneration. The healing time with CPS putty in extraction socket is found to be $4.9( \pm 0.8)$ months. ${ }^{10}$ PRF was used here as a GBR membrane to cover the graft material. $\mathrm{PRF}$ is an autologous platelet concentrate that encapsulates growth factor, cytokines and leukocytes in a fibrin matrix and released for a period of 7 days. The use of the PRF membranes may accelerate the integration and remodelling of the grafted biomaterial. ${ }^{11-13}$ PRF membranes have also been proven to be more suitable for in vitro cultivation of periosteal cells for bone tissue engineering compared to commercially available collagen membranes. ${ }^{14}$ Socket preservation helped in bone gain which can be used in the future as a site for prosthetic driven implant placement even if some complication occurs with the hemisection procedure.

\section{Conclusion}

The hemisection is a technique sensitive procedure and judicious case selection is must. Prognosis as good as any conventional endodontic or restorative procedure can be expected if performed correctly. It may be a suitable alternative to extraction and implant therapy and should be discussed with patients during consideration of treatment options. Socket preservation helped in bone gain which can be used in the future as a site for prosthetic driven implant placement even if some complication occurs with the hemisection procedure. In the present case, conservative approach was useful for successful preservation of the hopeless tooth along with future implant site development. A guiding principle should be to try and maintain what is present. 


\section{Acknowledgment}

None.

\section{Conflict of interest}

None.

\section{References}

1. Kost WJ, Stakiw JE. Root amputation and hemisection. J Can Dent Assoc. 1991;57(1):42-5.

2. Kurtzman GM, Silverstein LH, Shatz PC. Hemisection as an alternative treatment for vertically fractured mandibular molars. Compend Contin Educ Dent. 2006;27(2):126.

3. Basaraba N. Root amputation and tooth hemisection. Dent Clin North Am. 1969;13:121-32.

4. Weine FS. Endodontic Therapy. 5th ed, St. Louis: Mosby;1996.

5. Verma PK, Srivastava R, Gupta KK, et al. Combined endodonticperiodontal lesion: A clinical dilemma. J Interdiscip Dentistry. 2011;1(2):119-24.

6. Rotstein I, Simon JH. Diagnosis, prognosis and decision-making in the treatment of combined periodontal-endodontic lesions. Periodontol 2000. 2004;34:165-203.

7. Verma PK, Srivastava R, Baranwal HC, et al. A ray of hope for the hopeless: Hemisection of mandibular molar with socket preservation. Dent Hypotheses. 2012;3:159-63.
8. Bühler H. Survival rates of hemisected teeth: an attempt to compare them with survival rates of alloplastic implants. Int J Periodontics Restorative Dent. 1994;14(6):536-43.

9. Agrawal VS, Kapoor S, Shah NC. An innovative approach for treating vertically fractured mandibular molar-hemisection with socket preservation. J Interdiscip Dentistry. 2012;2(2):141-3.

10. Mahesh L, Salama MA, Kurtzman GM, et al. Socket grafting with calcium phosphosilicate alloplast putty: a histomorphometric evaluation. Compend Contin Educ Dent. 2012;33(8):e109-15.

11. Simonpieri A, Del Corso M, Sammartino G, et al. The relevance of Choukroun's platelet-rich fibrin and metronidazole during complex maxillary rehabilitations using bone allograft. Part II: Implant surgery, prosthodontics, and survival. Implant Dent. 2009;18(3):220-29.

12. Choukroun J, Diss A, Simonpieri A, et al. Platelet-rich fibrin (PRF): A second-generation platelet concentrate (Part IV): Clinical effects on tissue healing. Oral Surg Oral Med Oral Pathol Oral Radiol Endod. 2006;101(3):56-60.

13. Pradeep K, Kudva A, Narayanamoorthy V, et al. Platelet-rich fibrin combined with synthetic nanocrystalline hydroxy apatite granules in the management of radicular cyst. Niger J Clin Pract. 2016;19(5):688-91.

14. Gassling V, Douglas T, Warnke PH, et al. Platelet-rich fibrin membranes as scaffolds for periosteal tissue engineering. Clin Oral Implants Res. 2010;21(5):543-49. 